This item was submitted to Loughborough's Research Repository by the author.

Items in Figshare are protected by copyright, with all rights reserved, unless otherwise indicated.

The characteristic initial value problem for colliding plane waves: the linear case

PLEASE CITE THE PUBLISHED VERSION

LICENCE

CC BY-NC-ND 4.0

REPOSITORY RECORD

Griffiths, J.B., and Miguel Santano-Roco. 2019. "The Characteristic Initial Value Problem for Colliding Plane Waves: The Linear Case”. figshare. https://hdl.handle.net/2134/459. 


\title{
The characteristic initial value problem for colliding plane waves: The linear case
}

\author{
J. B. Griffiths* and M. Santano-Roco \\ Department of Mathematical Sciences, Loughborough University, \\ Loughborough, Leics. LE11 3TU, U.K.
}

June 24, 2002

\begin{abstract}
The physical situation of the collision and subsequent interaction of plane gravitational waves in a Minkowski background gives rise to a well-posed characteristic initial value problem in which initial data are specified on the two null characteristics that define the wavefronts. In this paper, we analyse how the Abel transform method can be used in practice to solve this problem for the linear case in which the polarization of the two gravitational waves is constant and aligned. We show how the method works for some known solutions, where problems arise in other cases, and how the problem can always be solved in terms of an infinite series if the spectral functions for the initial data can be evaluated explicitly.
\end{abstract}

\section{Introduction}

Many classes of explicit exact solutions are now known which model the collision and subsequent interaction between plane gravitational waves with distinct wavefronts which propagate into a Minkowski background (for a review see [1]). These solutions have invariably been obtained using an indirect approach which starts by finding a family of solutions of the field equations in the interaction region. The arbitrary parameters characterising the family are then restricted to specific ranges in order to satisfy the appropriate junction conditions for colliding plane waves. Finally, the restricted class of solutions obtained is extended backwards to determine the initial approaching waves that give rise to that particular family of solutions. This approach has enabled the general structure of this class of solutions to be understood. However, little progress has been made using the direct approach in which the approaching plane waves are specified initially and the subsequent interaction between them following their collision is then determined by solving the associated characteristic initial value problem.

It is known [2]-[3] that, in the vacuum (linear) case in which the approaching waves have constant and aligned polarization, the characteristic initial value problem may be solved theoretically using Riemann's method. Unfortunately, this method involves integrals which cannot be evaluated explicitly for appropriate initial data. As an alternative, Hauser and Ernst [4] have formulated a different method for solving the initial value problem in the linear case by making use of Abel transforms. Unfortunately, neither of these methods can be obviously generalised to the nonlinear case. However, some progress in treating this general case has recently been achieved. Hauser and Ernst [5]-[7] reformulated the corresponding nonlinear (vacuum) problem as a homogeneous Hilbert problem in a complex plane. Using this, they have proved that a solution of this problem exists, but their method has still not been used in practice to determine an explicit solution. However, another approach to boundary or initial value problems,

${ }^{*}$ E-mail: J.B.Griffiths@Lboro.ac.uk 
extended to include the electromagnetic case, had previously been formulated [8], [9] in terms of the monodromy transform method. Unfortunately, problems involving singularities at the point of collision are encountered when applying this method to the case of colliding plane waves. Nevertheless, these problems can be overcome. By extending the method to include dynamical monodromy data, a new method for solving the initial value problem has been achieved. This has been outlined in [10] and is described in detail in [11].

In the present work we concentrate on the linear case in which the polarization of the two initial gravitational waves is constant and aligned. We formulate the characteristic initial value problem for this situation in detail. We then demonstrate how the Abel transform method can be implemented in practice by deriving some well known solutions. Of course, to implement the Abel transform method to determine an explicit exact solution in the interaction region for given initial data, it is necessary to evaluate a number of integrals. We describe the particular difficulties that arise in attempting to implement this method for some physically interesting initial data. Finally, we demonstrate how the exact solution in the interaction region corresponding to arbitrary initial data can be constructed in terms of an infinite series involving hypergeometric functions.

\section{A collision of gravitational plane waves}

First, we recall that a plane gravitational wave with linear polarization and arbitrary profile $h(u)$ can be described by a metric in the Brinkmann form

$$
\mathrm{d} s^{2}=2 \mathrm{~d} u \mathrm{~d} r-\mathrm{d} X^{2}-\mathrm{d} Y^{2}+h(u)\left(X^{2}-Y^{2}\right) \mathrm{d} u^{2} .
$$

The wave surfaces for this wave are given by $u=$ const. It may be noted that the profile function appears explicitly in the metric. This has a distinct disadvantage when the profile takes a distributional form as, for example, in a description of impulsive waves. For this and a variety of other reasons, it will be convenient to transform the metric (1) to the Rosen form

$$
\mathrm{d} s^{2}=2 \mathrm{~d} u \mathrm{~d} v-P^{2} \mathrm{~d} x^{2}-Q^{2} \mathrm{~d} y^{2},
$$

which is always continuous, and involves two null coordinates $u$ and $v$. This can be achieved using the transformation $X=P(u) x, Y=Q(u) y, \quad r=v+\frac{1}{2} P P^{\prime} x^{2}+\frac{1}{2} Q Q^{\prime} y^{2}$, where

$$
P^{\prime \prime}+h(u) P=0 \quad \text { and } \quad Q^{\prime \prime}-h(u) Q=0,
$$

so that the only field equation is

$$
\frac{P^{\prime \prime}}{P}+\frac{Q^{\prime \prime}}{Q}=0
$$

Using a natural tetrad, the only non-zero component of the Weyl tensor for the metric (2) is given by

$$
\Psi_{4}=\frac{1}{2}\left(\frac{Q^{\prime \prime}}{Q}-\frac{P^{\prime \prime}}{P}\right)=h(u) .
$$

Now consider a gravitational wave (with constant polarization) which has a distinct wavefront. We can label the wavefront by $u=0$, so that $h(u)=0$ and the space is Minkowski for $u<0$. The wave may then be considered to have arbitrary profile $h(u)$ for $u \geq 0$, and be represented by the line element (2). We can also consider a second gravitational wave of a similar type to propagate into the initial Minkowski space from the opposite spatial direction. This may be considered to have a wavefront on the null hypersurface $v=0$ using the same notation as in (2). If this wave also has constant polarization (with the polarizations of the two waves aligned), the region $u<0, v \geq 0$ can also be described by a metric of the form (2) but with $P$ and $Q$ being functions of $v$ instead of $u$. 
In this way, we have set up initial conditions for the collision of two plane waves with constant aligned polarization and arbitrary profiles following the wavefronts. The space-time contains four distinct regions - a background Minkowski region $\mathrm{I}(u<0, v<0)$ which possesses the metric

$$
\mathrm{d} s^{2}=2 \mathrm{~d} u \mathrm{~d} v-\mathrm{d} x^{2}-\mathrm{d} y^{2},
$$

two wave regions II and III ( $u \geq 0, v<0$ and $u<0, v \geq 0$ respectively), and a region IV ( $u \geq 0$, $v \geq 0$ ) which is subsequent to the collision of the two waves and in which they interact. The general structure of such space-times is well known and illustrated in figure 1.



Figure 1: The structure of colliding plane wave space-times. Region I is the background Minkowski space, regions II and III contain the approaching plane waves, and region IV represents the interaction region following the collision. The singularity structure following the collision is known from the study of families of exact solutions and is described in [1].

For convenience, we will denote functions and parameters associated with the region II with the subscript + , and functions and parameters associated with the region III with the subscript - . Thus, the two waves which propagate into the background have profile functions $\Psi_{4}=h_{+}(u)$ for $u \geq 0, v<0$, and $\Psi_{0}=h_{-}(v)$ for $v \geq 0, u<0$.

The problem we now address is to initially specify the initial profile functions $h_{+}(u)$ and $h_{-}(v)$ and then to attempt to determine the metric to which this gives rise in the interaction region IV. This is a well posed characteristic initial value problem for which a unique solution is known to exist. However, as will be seen, it is not a simple matter to construct the solution explicitly.

\section{Analysis of the initial data}

It is found to be convenient to continue to use two future-pointing null coordinates $u$ and $v$ throughout the space-time, with the four distinct regions being separated by two null hypersurfaces $u=0$ and $v=0$ which represent the wavefronts of the approaching waves. The background region I $(u<0, v<0)$ ahead of each wave is taken to be part of Minkowski space with the metric (6).

Region II $(u \geq 0, v<0)$ contains one of the approaching plane waves. It is found to be convenient to represent this by the line element

$$
\mathrm{d} s^{2}=2 \mathrm{~d} u \mathrm{~d} v-e^{-U_{+}}\left(e^{V_{+}} \mathrm{d} x^{2}+e^{-V_{+}} \mathrm{d} y^{2}\right)
$$

in which the metric functions $U_{+}=-\log P_{+}-\log Q_{+}$, and $V_{+}=\log P_{+}-\log Q_{+}$depend on $u$ only. For any given initial profile function $h_{+}(u)$, they are constructed from the corresponding 
solutions of (3), and must satisfy the single equation

$$
2 U_{+, u u}=U_{+, u}^{2}+V_{+, u}^{2}
$$

which corresponds to (4). The amplitude of the initial gravitational wave is given by

$$
h_{+}(u)=-\frac{1}{2}\left(V_{+, u u}-U_{+, u} V_{+, u}\right)
$$

In order to join this region smoothly with the metric (6) across the null hypersurface $u=0$, without introducing impulsive components in the Ricci tensor, it is necessary that $U_{+}(0)=0$, $U_{+, u}(0)=0$, and $V_{+}(0)=0$. It is then clear from (8) that $U_{+}$and $U_{+, u}$ must be monotonically increasing functions. Hence $e^{-U_{+}}$is a monotonically decreasing function in this region and can be expanded in the form

$$
e^{-U_{+}}=1-\left(c_{+} u\right)^{n_{+}}+o\left(u^{n_{+}}\right)
$$

near the wavefront $u=0$, where $c_{+}>0$ and $n_{+} \geq 2$ are constants. It then follows from (8) that, near the wavefront, $V_{+}$must be such that

$$
V_{+, u}^{2}=2 n_{+}\left(n_{+}-1\right) c_{+}^{n_{+}} u^{\left(n_{+}-2\right)}+o\left(u^{\left(n_{+}-2\right)}\right),
$$

and the amplitude function behaves as

$$
h_{+}(u)= \pm \frac{1}{4} \sqrt{2 n_{+}\left(n_{+}-1\right)}\left(n_{+}-2\right) c_{+}^{n_{+} / 2} u^{\left(n_{+} / 2\right)-2}+o\left(u^{\left(n_{+} / 2\right)-2}\right),
$$

where the sign is opposite to that of $V_{+, u}$. (It is assumed that all functions are sufficiently smooth as $u \rightarrow 0$.) It follows from this that the parameters $n_{+}$and $c_{+}$are determined uniquely from the initial data - specifically from the behaviour of $h_{+}(u)$ near the wavefront. (The case when $n_{+}=2$ generally contains an impulsive component on the wavefront $u=0$, and needs to be treated a little more carefully.)

Now, it is always possible to relabel the null hypersurfaces using an arbitrary $C^{2}$ transformation $u \rightarrow \tilde{u}=\tilde{u}(u)$. In practice, this freedom is almost always required in the construction of explicit solutions. However, it has the effect of replacing the term $2 \mathrm{~d} u \mathrm{~d} v$ in the line element (7) by $2 e^{-M_{+}(u)} \mathrm{d} u \mathrm{~d} v$. Further, in order to avoid introducing a coordinate singularity and to maintain continuity with the background metric in the form (6), it is necessary that $\mathrm{d} u / \mathrm{d} \tilde{u}=1$ on the wavefront labelled $u=0, \tilde{u}=0$. Using this freedom, it is possible to choose some particular form for $e^{-U_{+}}$, such as

$$
e^{-U_{+}}=1-\left(c_{+} u\right)^{n_{+}} .
$$

It is significant that the power of the leading term in the expansion of the metric function $U_{+}$cannot be changed. i.e. $n_{+}$is an essential parameter which is determined by the initial data. Physically, this parameter determines the character of the wavefront; mathematically, it determines the degree of continuity of $U_{+}$on the wavefront $u=0$.

Retaining complete generality at this stage it is convenient to put

$$
e^{-U_{+}}=\frac{1}{2}+f(u)
$$

and then to use $f$ as a coordinate in place of $u$. It may be observed that this function is given by $f=P_{+} Q_{+}-\frac{1}{2}$, which is explicitly determined by the initial data. To maintain the character of the wavefront at $u=0$, it is necessary that $f(u)$ behaves as

$$
f(u)=\frac{1}{2}-\left(c_{+} u\right)^{n_{+}}+o\left(u^{n_{+}}\right)
$$

near the wavefront. In fact it is always possible (although not always convenient) to use the above coordinate freedom to introduce a special gauge (13) such that

$$
f(u)=\frac{1}{2}-\left(c_{+} u\right)^{n_{+}} .
$$


The additional metric function $M_{+}$, which is normally introduced with any special choice of gauge, is then determined by the equation

$$
M_{+f}=-\frac{f^{\prime \prime}}{f^{\prime 2}}+\frac{1}{2\left(f+\frac{1}{2}\right)}-\frac{1}{2}\left(f+\frac{1}{2}\right) V_{+f^{2}},
$$

which replaces (8). This equation is critical in the colliding plane wave problem as it contains a singular term on the wavefront on which $f^{\prime}=0$, and this must be counterbalanced by an appropriate singular behaviour in the function $V_{+}$. In order for $M_{+}$to be continuous across the boundary $u=0$, on which $f^{\prime}=0$, it is necessary that $V_{+}$satisfies (11). Rewritten in terms of the function $f$, this condition can be expressed as

$$
\lim _{f \rightarrow 1 / 2}\left[\left(\frac{1}{2}-f\right)\left(V_{+f}\right)^{2}\right]=2 k_{+},
$$

where $k_{+}=1-1 / n_{+}$i.e.

$$
\frac{1}{2} \leq k_{+}<1 .
$$

Region III $(u<0, v \geq 0)$, contains the other approaching plane gravitational wave with the same (i.e. aligned) polarization. The metric can be taken in the same form as that for region II but with the roles of $u$ and $v$ reversed. It is here convenient to put

$$
e^{-U_{-}}=\frac{1}{2}+g(v),
$$

and to use $g$ as a coordinate in place of $v$. Again, it is sometimes convenient to adopt a gauge such that

$$
g(v)=\frac{1}{2}-\left(c_{-} v\right)^{n_{-}} .
$$

It may be noted that the amplitude of the two waves and the character of the wavefronts are determined by the constants $c_{+}, c_{-}, n_{+}$and $n_{-}$. The latter two are fixed by the initial data, but it is always possible to make a boost $u \rightarrow c \tilde{u}, v \rightarrow c^{-1} \tilde{v}$ such that either $c_{+}$or $c_{-}$can be made equal to unity. (Alternatively, they could be made equal.)

The gravitational wave in this region is then specified by the functions $g, V_{-}(g)$ and $M_{-}(g)$ which are again related by a single equation corresponding to (16), and by the single parameter $n_{-} \geq 2$ which is contained in the form of $g(v)$. To set consistent initial conditions (and to ensure that $M_{-}$is at least $C^{0}$ across the wavefront), it is necessary that the functions satisfy the condition

$$
\lim _{g \rightarrow 1 / 2}\left[\left(\frac{1}{2}-g\right)\left(V_{-g}\right)^{2}\right]=2 k_{-},
$$

where $\frac{1}{2} \leq k_{-}<1$, (or $\left.k_{-}=1-1 / n_{-}\right)$.

It is always possible to choose initial $x, y$ coordinates such that the metric on the wavefronts prior to the collision is continuous with the background metric in the form (6). It is therefore appropriate to impose the additional initial conditions that $V_{+}\left(\frac{1}{2}\right)=0, V_{-}\left(\frac{1}{2}\right)=0, M_{+}\left(\frac{1}{2}\right)=0$ and $M_{-}\left(\frac{1}{2}\right)=0$.

Region IV $(u \geq 0, v \geq 0)$ : In the vacuum case for the collision of plane gravitational waves with constant aligned polarization, this region can always be described by the line element

$$
\mathrm{d} s^{2}=2 e^{-M} \mathrm{~d} u \mathrm{~d} v-e^{-U}\left(e^{V} \mathrm{~d} x^{2}+e^{-V} \mathrm{~d} y^{2}\right)
$$

where $U, V$ and $M$ are functions of both $u$ and $v$. The field equations immediately imply that $e^{-U}$ satisfies a wave equation, so that we can put

$$
e^{-U}=f(u)+g(v),
$$

where $f(u)$ and $g(v)$ are arbitrary functions subject to $f+g>0$. However, since $e^{-U}$ must be continuous across the boundaries $u=0$ and $v=0, f(u)$ and $g(v)$ must retain the same forms as 
have already been prescribed in the initial regions. If the special gauge (15) and (19) is adopted, it is possible to make the global definitions

$$
f(u)=\frac{1}{2}-\left(c_{+} u\right)^{n_{+}} \Theta(u), \quad g(v)=\frac{1}{2}-\left(c_{-} v\right)^{n_{-}} \Theta(v)
$$

where $\Theta(u)$ is the Heaviside step function, but this prescription is not essential (and is often not convenient).

It may immediately be observed that a singularity of some kind will occur in the interaction region on the spacelike hypersurface on which $f+g=0$. It can be shown (see references in [1]) that this is generically a curvature singularity, although families of solutions with infinitely many parameters exist in which it corresponds to an unstable Cauchy horizon. It is referred to as a "focussing" singularity in figure 1.

In complete generality, we can adopt $f$ and $g$ as coordinates throughout the interaction region, in which $f<\frac{1}{2}, g<\frac{1}{2}$ and $f+g>0$. With this, the main vacuum field equation is

$$
(f+g) V_{f g}+\frac{1}{2} V_{f}+\frac{1}{2} V_{g}=0,
$$

which is an Euler-Poisson-Darboux equation with non-integer coefficients. For any solution of this equation, $M$ can be obtained, at least in principle, by integrating the remaining field equations

$$
\begin{aligned}
& M_{f}=-\frac{f^{\prime \prime}}{f^{\prime 2}}+\frac{1}{2(f+g)}-\frac{1}{2}(f+g) V_{f}^{2} \\
& M_{g}=-\frac{g^{\prime \prime}}{g^{\prime 2}}+\frac{1}{2(f+g)}-\frac{1}{2}(f+g) V_{g}^{2}
\end{aligned}
$$

In this case, the initial data is defined by the functions $f, g, V_{+}(f)$ and $V_{-}(g)$ and the solution of (24) in the interaction region $V(f, g)$ must satisfy

$$
V\left(f, \frac{1}{2}\right)=V_{+}(f), \quad V\left(\frac{1}{2}, g\right)=V_{-}(g) .
$$

with $V\left(\frac{1}{2}, \frac{1}{2}\right)=0$. If these conditions are satisfied, the required continuity of $M$ is assured. With given initial data, these conditions are sufficient to uniquely determine the solution of the colliding plane wave problem in the interaction region, at least up to the focussing singularity. We recall that, for colliding plane waves, the metric functions must be continuous across the wavefronts, while $V_{f}$ must be unbounded on $f=\frac{1}{2}$ but constrained by (17). Similarly, $V_{g}$ must be unbounded on $g=\frac{1}{2}$ subject to (20). However, these conditions are already included in the initial data functions $V_{+}(f)$ and $V_{-}(g)$.

\section{The Abel transform solution to the initial value problem}

A separable solution of equation (24) can easily be obtained in the form

$$
V(f, g)=A(\sigma-f)^{-\frac{1}{2}}(\sigma+g)^{-\frac{1}{2}}
$$

where $A$ and $\sigma$ are arbitrary constants. Since many such functions can be superposed, we can replace $A$ by $A(\sigma) \sqrt{\sigma+\frac{1}{2}}$ and treat $\sigma$ as a "spectral" parameter and $A(\sigma)$ as a spectral amplitude function. Introducing also a second function $B(\sigma)$, a general class of solutions for the interaction region IV can be expressed in the form

$$
V(f, g)=\int_{f}^{\frac{1}{2}} \frac{A(\sigma) \sqrt{\sigma+\frac{1}{2}}}{\sqrt{\sigma-f} \sqrt{\sigma+g}} \mathrm{~d} \sigma+\int_{g}^{\frac{1}{2}} \frac{B(\sigma) \sqrt{\sigma+\frac{1}{2}}}{\sqrt{\sigma+f} \sqrt{\sigma-g}} \mathrm{~d} \sigma .
$$

By considering the limits of this arbitrarily close to the two wavefronts as $g \rightarrow \frac{1}{2}$ and $f \rightarrow \frac{1}{2}$, it can be seen that this solution is consistent with the initial data provided

$$
V_{+}(f)=\int_{f}^{\frac{1}{2}} \frac{A(\sigma)}{\sqrt{\sigma-f}} \mathrm{~d} \sigma, \quad V_{-}(g)=\int_{g}^{\frac{1}{2}} \frac{B(\sigma)}{\sqrt{\sigma-g}} \mathrm{~d} \sigma .
$$


As observed by Hauser and Ernst [4], these integrals are exactly those that define the Abel transform. It then follows that the spectral amplitude functions $A(\sigma)$ and $B(\sigma)$ can be determined explicitly from the initial data functions $V_{+}(f)$ and $V_{-}(g)$ using the inverse Abel transform

$$
A(\sigma)=-\frac{1}{\pi} \int_{\sigma}^{\frac{1}{2}} \frac{V_{+}^{\prime}(f)}{\sqrt{f-\sigma}} \mathrm{d} f, \quad B(\sigma)=-\frac{1}{\pi} \int_{\sigma}^{\frac{1}{2}} \frac{V_{-}^{\prime}(g)}{\sqrt{g-\sigma}} \mathrm{d} g .
$$

When comparing (30) with (29), it may be observed that the solution in the interaction region involves the introduction of a dependence upon $g$ in the integral involving $A(\sigma)$ and a dependence upon $f$ in the integral involving $B(\sigma)$. This is an initial indication of a kind of "dynamical monodromy data" (as will be required for the nonlinear case and explained in [10] and [11]) in which initial spectral data on one characteristic is modified by the presence of that on the other.

Given initial data in the form of the specified functions $f, g, V_{+}(f)$ and $V_{-}(g)$, the derivatives $V_{+}^{\prime}(f)$ and $V_{-}^{\prime}(g)$ can be computed and substituted into (31). The spectral functions $A(\sigma)$ and $B(\sigma)$ are then uniquely determined, and the solution throughout the interaction region is given by (29). This solves the initial value problem for the linear case, at least theoretically. In practice, it depends on whether or not the integrals for $A(\sigma)$ and $B(\sigma)$ in $(31)$ and $V(f, g)$ in (29) can be explicitly evaluated.

It is possible that $V_{+}(f)$ and $V_{-}(g)$ may be expressed as a sum of distinct terms. It may then be observed that the Abel transform method (29)-(31) is linear. Thus, each component of $V_{ \pm}$ will give rise to separate components of the spectral functions $A(\sigma)$ and $B(\sigma)$. These different components will give rise to different components of the solution in the interaction region. Since the main field equation (24) is also linear, the complete solution may be obtained as a simple sum over all these components.

Of course it is not necessary to construct the solution in the interaction region using a single integral. In cases in which the expressions change over different intervals (e.g. for sandwich waves, or waves with successive impulsive components), it is appropriate to divide the interaction region into a number of sub-regions and to determine the solution in each region successively. Initial data can be specified on any pair of null characteristics. Starting from any particular wavefront given by $f=f_{1}$, the Abel transform given above can immediately be generalised to

$$
V_{+}(f)=V_{+}\left(f_{1}\right)+\int_{f}^{f_{1}} \frac{A(\sigma)}{\sqrt{\sigma-f}} \mathrm{~d} \sigma, \quad A(\sigma)=-\frac{1}{\pi} \int_{\sigma}^{f_{1}} \frac{V_{+}^{\prime}(f)}{\sqrt{f-\sigma}} \mathrm{d} f .
$$

This enables the Abel transform method to be used in a sequence of regions.

In particular, the solution of the characteristic initial value problem, in which data are given on the two characteristics $f=f_{1}$ and $g=g_{1}$, is given by first determining the spectral data

$$
A(\sigma)=-\frac{1}{\pi} \int_{\sigma}^{f_{1}} \frac{V_{+}^{\prime}\left(f, g_{1}\right)}{\sqrt{f-\sigma}} \mathrm{d} f, \quad B(\sigma)=-\frac{1}{\pi} \int_{\sigma}^{g_{1}} \frac{V_{-}^{\prime}\left(f_{1}, g\right)}{\sqrt{g-\sigma}} \mathrm{d} g .
$$

The solution can then be obtained by evaluating

$$
V(f, g)=V\left(f_{1}, g_{1}\right)+\int_{f}^{f_{1}} \frac{A(\sigma)}{\sqrt{\sigma-f}} \frac{\sqrt{\sigma+g_{1}}}{\sqrt{\sigma+g}} \mathrm{~d} \sigma+\int_{g}^{g_{1}} \frac{B(\sigma)}{\sqrt{\sigma-g}} \frac{\sqrt{\sigma+f_{1}}}{\sqrt{\sigma+f}} \mathrm{~d} \sigma .
$$

\section{Illustrations of the Abel transform method}

In this section, we will illustrate how the above method can be used explicitly to construct some specific solutions.

As a first example, we will derive the Khan-Penrose solution [12] which describes the collision of impulsive gravitational waves with aligned polarization. In this case, $h_{+}(u)=\delta(u)$ so that $P_{+}=1-u \Theta(u), Q_{+}=1+u \Theta(u)$, and the solution in region II is given by

$$
e^{-U_{+}}=1-u^{2}, \quad e^{V_{+}}=\frac{1-u}{1+u} .
$$


Thus $n_{+}=2$, and we may put

$$
f=\frac{1}{2}-u^{2}, \quad \text { and } \quad V_{+}(f)=\log \left(1-\sqrt{\frac{1}{2}-f}\right)-\log \left(1+\sqrt{\frac{1}{2}-f}\right) .
$$

Similarly in region III, $h_{-}(v)=\delta(v)$, and the solution is given by

$$
e^{-U_{-}}=1-v^{2}, \quad e^{V_{-}}=\frac{1-v}{1+v}
$$

so that $n_{-}=2$, and we can put

$$
g=\frac{1}{2}-v^{2}, \quad \text { and } \quad V_{-}(g)=\log \left(1-\sqrt{\frac{1}{2}-g}\right)-\log \left(1+\sqrt{\frac{1}{2}-g}\right) .
$$

We may now use the Abel transform to determine the "spectral" functions $A(\sigma)$ and $B(\sigma)$ that are required in the above solution of the (linear) initial value problem. In this case, using initial data on the null characteristic $v=0, u \geq 0$, we obtain

$$
\begin{aligned}
A(\sigma) & =-\frac{1}{\pi} \int_{\sigma}^{\frac{1}{2}} \frac{1}{\sqrt{f-\sigma} \sqrt{\frac{1}{2}-f}\left(\frac{1}{2}+f\right)} \mathrm{d} f \\
& =\frac{1}{\pi}\left[\frac{2}{\sqrt{\frac{1}{2}+\sigma}} \tan ^{-1}\left(\sqrt{\frac{1}{2}+\sigma} \frac{\sqrt{\frac{1}{2}-f}}{\sqrt{f-\sigma}}\right)\right]_{\sigma}^{\frac{1}{2}} \\
& =-\left(\frac{1}{2}+\sigma\right)^{-1 / 2}
\end{aligned}
$$

By the identical calculation, using the initial data on the other null characteristic $u=0, v \geq 0$, we obtain similarly that $B(\sigma)=-\left(\frac{1}{2}+\sigma\right)^{-1 / 2}$.

The solution in the interaction region may finally be obtained by evaluating

$$
\begin{aligned}
V(f, g)= & -\int_{f}^{\frac{1}{2}} \frac{1}{\sqrt{\sigma-f} \sqrt{\sigma+g}} \mathrm{~d} \sigma-\int_{g}^{\frac{1}{2}} \frac{1}{\sqrt{\sigma+f} \sqrt{\sigma-g}} \mathrm{~d} \sigma \\
= & -[\log (2 \sigma-f+g+2 \sqrt{\sigma-f} \sqrt{\sigma+g})]_{f}^{\frac{1}{2}} \\
& \quad-[\log (2 \sigma+f-g+2 \sqrt{\sigma+f} \sqrt{\sigma-g})]_{g}^{\frac{1}{2}} \\
= & \log \left(\frac{\sqrt{\frac{1}{2}+g}-\sqrt{\frac{1}{2}-f}}{\sqrt{\frac{1}{2}+g}+\sqrt{\frac{1}{2}-f}}\right)+\log \left(\frac{\sqrt{\frac{1}{2}+f}-\sqrt{\frac{1}{2}-g}}{\sqrt{\frac{1}{2}+f}+\sqrt{\frac{1}{2}-g}}\right)
\end{aligned}
$$

This is exactly the Khan-Penrose solution. To obtain the complete solution, it only remains to integrate the subsidiary equations (25) and (26) to obtain the required expression for $M$.

The above example is a particularly important illustration of the method for constructing explicit solutions for given initial data since it describes the collision of impulsive gravitational waves. For such waves, the metric possesses the lowest possible degree of continuity across the wavefronts. In this sense, the above solution represents the "worst case", and it is important to observe that the above Abel transform method works perfectly adequately.

The above solution can immediately be generalised to that of Szekeres [2] in which $n_{ \pm} \geq 2$ simply by multiplying $V_{+}(f)$ and $V_{-}(g)$, and hence $A(\sigma)$ and $B(\sigma)$, by the constants $\sqrt{2 k_{+}}$ and $\sqrt{2 k_{-}}$respectively. For this case the two terms in (40) are multiplied by $\sqrt{2 k_{+}}$and $\sqrt{2 k_{-}}$ respectively. However, for this solution, the initial profiles are given by

$$
h_{+}(u)=\left\{\begin{array}{cc}
\delta(u) & \text { if } n_{+}=2 \\
\frac{\sqrt{n_{+}\left(n_{+}-1\right)}\left(n_{+}-2\right)}{2 \sqrt{2}} \frac{u^{\left(n_{+} / 2\right)-2}}{\left(1-u^{\left.n_{+}\right)^{3-\left(2 / n_{+}\right)}} \Theta(u)\right.} & \text { if } n_{+}>2
\end{array}\right.
$$


with equivalent expressions for $h_{-}(v)$. These have the unphysical property that, apart from the Khan-Penrose case, the wave amplitude becomes unbounded in the initial regions as $u \rightarrow 1$, or $f \rightarrow-\frac{1}{2}$.

The above example is a member of a family of solutions of (24) that can be expressed as products of Legendre functions. For these it is convenient to introduce new coordinates $t$ and $z$ defined by

$$
\begin{aligned}
t & =\sqrt{\frac{1}{2}-f} \sqrt{\frac{1}{2}+g}+\sqrt{\frac{1}{2}-g} \sqrt{\frac{1}{2}+f} \\
z & =\sqrt{\frac{1}{2}-f} \sqrt{\frac{1}{2}+g}-\sqrt{\frac{1}{2}-g} \sqrt{\frac{1}{2}+f} .
\end{aligned}
$$

The general family of solutions is then expressed as the sum

$$
V=\sum_{n}\left(a_{n} P_{n}(t) P_{n}(z)+q_{n} Q_{n}(t) P_{n}(z)+p_{n} P_{n}(t) Q_{n}(z)+b_{n} Q_{n}(t) Q_{n}(z)\right)
$$

where $P_{n}(x)$ and $Q_{n}(x)$ are Legendre functions of the first and second kinds respectively, and $a_{n}, q_{n}, p_{n}$ and $b_{n}$ are series of arbitrary constants. For example, the Szekeres solution is given by $V=-\sqrt{2}\left(\sqrt{k}_{+}+\sqrt{k}_{-}\right) Q_{0}(t) P_{0}(z)-\sqrt{2}\left(\sqrt{k}_{+}-\sqrt{k}_{-}\right) P_{0}(t) Q_{0}(z)$, which also includes the Khan-Penrose solution. To satisfy the colliding plane wave conditions (17) and (20), which require that the derivative of $V$ with respect to $f$ and $g$ be unbounded on the wavefront, it is necessary that at least one Legendre function of the second kind be included.

When considering the initial data that gives rise to these solutions, the associated spectral functions can readily be calculated, and the solution corresponding to each component can be determined, at least in the simpler cases. For example, the spectral functions for the initial data associated with the following components are:

$$
\begin{array}{lll}
V=Q_{0}(t) P_{0}(z): & A(\sigma)=\frac{1}{2 \sqrt{\frac{1}{2}+\sigma}}, & B(\sigma)=\frac{1}{2 \sqrt{\frac{1}{2}+\sigma}}, \\
V=Q_{1}(t) P_{1}(z): & A(\sigma)=-\frac{\sigma}{\sqrt{\frac{1}{2}+\sigma}}, & B(\sigma)=\frac{\sigma}{\sqrt{\frac{1}{2}+\sigma}}, \\
V=Q_{2}(t) P_{2}(z): & A(\sigma)=-\frac{12 \sigma^{2}-1}{4 \sqrt{\frac{1}{2}+\sigma}}, & B(\sigma)=-\frac{12 \sigma^{2}-1}{4 \sqrt{\frac{1}{2}+\sigma}}, \\
V=Q_{3}(t) P_{3}(z): & A(\sigma)=\frac{20 \sigma^{3}-3 \sigma}{2 \sqrt{\frac{1}{2}+\sigma}}, & B(\sigma)=-\frac{20 \sigma^{3}-3 \sigma}{2 \sqrt{\frac{1}{2}+\sigma}} .
\end{array}
$$

The complete solution corresponding to these functions can readily be evaluated. However, it may be observed that, for the same reasons as those outlined above, combinations of these solutions do not generally correspond to realistic initial data.

Another disadvantage of the expansion (43) is that the $f$ and $g$ derivatives of all the components which involve Legendre functions of the second kind are unbounded on the wavefronts. Thus, the structure of the wavefronts are represented by an unlimited number of these terms. All the coefficients appear in the conditions (17) and (20), and so are constrained by the two constants $n_{ \pm}$.

\section{Some difficulties which arise in practice}

It may be noted that, for sandwich waves or sequences of impulsive waves, $h_{+}(u)$ is nonzero over a short interval only. If we denote the end of this interval by $u=u_{1}$, we can consider the case in which $h_{+}(u)=0$ for $u>u_{1}$. Over this subsequent interval, (3) implies that $P_{+}(u)$ and $Q_{+}(u)$ must be linear in $u$, and it can be shown that the spectral function must take the form

$$
A(\sigma)=-\frac{2}{\pi \sqrt{\sigma+\frac{1}{2}}} \tan ^{-1}\left(c \frac{\sqrt{f_{1}-\sigma}}{\sqrt{\sigma+\frac{1}{2}}}\right)
$$


where $f_{1}=f\left(u_{1}\right)$, and $c$ is some constant. Although the initial data can be reconstructed from this expression using (30), the metric in the interaction region can only be evaluated explicitly using (29) for the case of the Khan-Penrose solution for which $c=\infty$. For all other cases, (29) gives rise to integrals of elliptic integrals of the third kind. For such situations it is most unlikely that the exact solution in the interaction region can be expressed using a finite number of expressions involving only elementary functions.

Using (8), it may be noted that the profile function (9) can be expressed in the form

$$
h_{+}(u)=-\frac{1}{2}\left(V_{+, u u}-U_{+, u u}\right)-\frac{1}{4}\left(V_{+, u}-U_{+, u}\right)^{2} .
$$

From this, it would appear to be natural to include $U_{+}$as one component of $V_{+}$. However, the spectral function for this component takes the form

$$
A(\sigma)=\frac{2}{\pi} \frac{1}{\sqrt{\frac{1}{2}+\sigma}} \tan ^{-1} \sqrt{\frac{\frac{1}{2}-\sigma}{\frac{1}{2}+\sigma}} .
$$

Although the initial data can be reconstructed from this expression, (29) again gives rise to integrals of elliptic integrals of the third kind which cannot be evaluated explicitly in terms of elementary functions. (The degenerate Ferrari-Ibañez solution [13], for which the interaction region is locally isometric to part of the Schwarzschild solution inside the horizon, is exactly of this form. It corresponds to the case in which $V_{ \pm}$is the sum of the Khan-Penrose expression (36) or $(38)$ and $U_{ \pm}$.)

It may be noted that (9) can also be expressed in the form

$$
h_{+}(u)=-\frac{1}{2} V_{+, u u}-\frac{f^{\prime} V_{+, u}}{2\left(f+\frac{1}{2}\right)}
$$

from which it is clear that, for most initial choices of $V_{+}(u), h_{+}(u)$ must be unbounded as $f \rightarrow-\frac{1}{2}$. This feature is indeed observed for almost all the "known" solutions for colliding plane waves (including the degenerate Ferrari-Ibañez solution). For the initial value problem considered here, however, it is considered preferable that this singularity in the initial data does not occur. We prefer to assume that realistic expressions for $V_{+}(u)$ should normally take a form in which such a singularity is avoided. However, apart from the Khan-Penrose solution, none of the known families of solutions of $(24)$ for $V_{+}(u)$ which satisfy the condition (17) also satisfy this regularity condition.

These observations would seem to argue that, for collisions of plane waves with reasonably realistic expressions for $h_{+}(u)$ and $h_{-}(v)$, it is most unlikely that exact solutions representing the interaction region will be obtainable in any explicit form involving elementary functions.

\section{Series of self-similar solutions}

We may, however, recall that there exists a family of self-similar solutions of (24) that have been described in [14]-[16] which represent waves with distinct wavefronts. These were originally presented in the context of different backgrounds, and in a form in which the wavefront $u=0$ is given by $f=0$. We will now demonstrate how this approach can be applied to the colliding plane wave problem.

In order to consider the wave with the wavefront $u=0, f=\frac{1}{2}$, we replace the coordinates $f$ and $g$ in [14]-[16] by $f-\frac{1}{2}$ and $g+\frac{1}{2}$ respectively. We then introduce new coordinates $\tau, \zeta$ in the interaction region defined by

$$
\tau=f+g, \quad \zeta=\frac{1-f+g}{f+g},
$$


In terms of the gauge (23), these take the form

$$
\tau=1-\left(c_{+} u\right)^{n_{+}}-\left(c_{-} v\right)^{n_{-}}, \quad \zeta=\frac{1+\left(c_{+} u\right)^{n_{+}}-\left(c_{-} v\right)^{n_{-}}}{1-\left(c_{+} u\right)^{n_{+}}-\left(c_{-} v\right)^{n_{-}}},
$$

although this restriction is not necessary. Using the new coordinates, the wavefront is given by $\zeta=1$, and a solution of (24) can be expressed in the self-similar form

$$
V(\tau, \zeta)=\tau^{k} H_{k}(\zeta)
$$

where $k$ is an arbitrary real (non-negative) parameter. The condition that $V=0$ on this wavefront is expressed by the constraint $H_{k}(1)=0$ for $k \geq 0$. By direct substitution, it is found that the functions $H_{k}(\zeta)$ must satisfy the linear ordinary differential equation

$$
\left(1-\zeta^{2}\right) H_{k}^{\prime \prime}+(2 k-1) \zeta H_{k}^{\prime}-k^{2} H_{k}=0 .
$$

With the above initial conditions, these functions satisfy the recursion relations

$$
H_{k}(\zeta)=\int_{1}^{\zeta} H_{k-1}\left(\zeta^{\prime}\right) d \zeta^{\prime} \quad \text { so that } \quad H_{k}^{\prime}(\zeta)=H_{k-1}(\zeta) .
$$

For integer values of $k$, explicit expressions for these solutions can be obtained in terms of elementary functions from the initial solution $H_{0}(\zeta)=\cosh ^{-1} \zeta$.

The solutions described above can be expressed in terms of standard hypergeometric functions $F(a, b ; c ; z)$ in the form

$$
(f+g)^{k} H_{k}\left(\frac{1-f+g}{f+g}\right)=c_{k} \frac{\left(\frac{1}{2}-f\right)^{1 / 2+k}}{\sqrt{f+g}} F\left(\frac{1}{2}, \frac{1}{2} ; \frac{3}{2}+k ; \frac{f-\frac{1}{2}}{f+g}\right)
$$

where, for integer $k$

$$
c_{k}=(-1)^{k} \frac{2^{k} \Gamma\left(\frac{3}{2}\right)}{\Gamma\left(k+\frac{3}{2}\right)} .
$$

When applying the above recurrence relation for arbitrary values of $k$, we only require the recursion relation $c_{k-1}=-\frac{1}{2}\left(k+\frac{1}{2}\right) c_{k}$.

It may be noted that the condition (17) on the wavefront is satisfied only for the case $k=0$, and then only with the additional multiplicative constant $a_{0}=2 \sqrt{2 k_{+}}$. However, in view of the linearity of this case, an arbitrary number of higher order terms may also be included. In addition, the equivalent solutions with a distinct wavefront which propagate in the opposite direction, and have the wavefront $v=0$ on which $g=\frac{1}{2}$, can also be included. In this way, a general solution in the interaction region can be expressed as

$$
V(f, g)=\sum_{k=0}^{\infty} a_{k}(f+g)^{k} H_{k}\left(\frac{1-f+g}{f+g}\right)+\sum_{k=0}^{\infty} b_{k}(f+g)^{k} H_{k}\left(\frac{1+f-g}{f+g}\right)
$$

where $a_{0}=2 \sqrt{2 k_{+}}$and $b_{0}=2 \sqrt{2 k_{-}}$. It may be observed that these series have the advantage that terms with higher values of $k$ have a higher degree of differentiability on the wavefront. This may be contrasted with other series representations (in terms of Legendre or Bessel functions etc.) in which all terms affect the differentiability properties on the wavefront. On the other hand, it may well not be possible to explicitly evaluate the quadratures for the remaining metric function $M$. Nevertheless, many terms can be evaluated using the methods described in [14]-[16] and some qualitative properties may be deduced.

The solution described above may be considered to be valid throughout the interaction region $f<\frac{1}{2}, g<\frac{1}{2}$ and $f+g>0$. It can then be extended back to region II, according to the 
Penrose construction, simply by setting $g=\frac{1}{2}$. The resulting expression must then coincide with that prescribed by the initial data. Thus,

$$
V\left(f, \frac{1}{2}\right)=\sum_{k=0}^{\infty} a_{k}\left(\frac{1}{2}+f\right)^{k} H_{k}\left(\frac{\frac{3}{2}-f}{\frac{1}{2}+f}\right)=V_{+}(f) .
$$

Similarly, on the junction with region III, we require

$$
V\left(\frac{1}{2}, g\right)=\sum_{k=0}^{\infty} b_{k}\left(\frac{1}{2}+g\right)^{k} H_{k}\left(\frac{\frac{3}{2}-g}{\frac{1}{2}+g}\right)=V_{-}(g) .
$$

The given initial data $V_{+}(f)$ and $V_{-}(g)$ can therefore be re-expressed in terms of two sequences of constants $a_{k}$ and $b_{k}$. However, to address the initial value problem, we need to find a way of determining the two sets of constants $a_{k}$ and $b_{k}$ from the initial data functions $V_{+}(f)$ and $V_{-}(g)$ respectively.

Once the coefficients $a_{k}$ and $b_{k}$ are known, the spectral functions associated with each component can be obtained by evaluating

$$
A_{k}(\sigma)=-\frac{1}{\pi} \int_{\sigma}^{\frac{1}{2}} \frac{V_{+k}^{\prime}(f)}{\sqrt{f-\sigma}} \mathrm{d} f, \quad \text { where } \quad V_{+k}(f)=a_{k}\left(\frac{1}{2}+f\right)^{k} H_{k}\left(\frac{\frac{3}{2}-f}{\frac{1}{2}+f}\right),
$$

and similar expressions for $B_{k}(\sigma)$ and $V_{-k}(g)$ involving the constant $b_{k}$. It can be seen, however, that the integral (59) must take the form

$$
A_{k}(\sigma)=p_{k}\left(\frac{1}{2}-\sigma\right)^{k}\left(\frac{1}{2}+\sigma\right)^{-1 / 2}
$$

where $p_{k}$ is a constant that is to be determined. This result has been obtained by substituting into the inverse transformation to reconstruct the original component. Moreover, there is no difficulty in including also an explicit $g$-dependence as required in the general expression (29). Explicitly, the component in the interaction region corresponding to $A_{k}(\sigma)$ is

$$
\begin{aligned}
V_{k}(f, g) & =p_{k} \int_{f}^{\frac{1}{2}} \frac{\left(\frac{1}{2}-\sigma\right)^{k}}{\sqrt{\sigma-f} \sqrt{\sigma+g}} \mathrm{~d} \sigma \\
& =p_{k} \frac{\left(\frac{1}{2}-f\right)^{\frac{1}{2}+k}}{\sqrt{f+g}} \int_{0}^{1} t^{-1 / 2}(1-t)^{k}\left(1+\left(\frac{\frac{1}{2}-f}{f+g}\right) t\right)^{-1 / 2} \mathrm{~d} t \\
& =p_{k} \frac{\Gamma\left(\frac{1}{2}\right) \Gamma(1+k)}{\Gamma\left(\frac{3}{2}+k\right)} \frac{\left(\frac{1}{2}-f\right)^{\frac{1}{2}+k}}{\sqrt{f+g}} F\left(\frac{1}{2}, \frac{1}{2} ; \frac{3}{2}+k ;-\frac{\frac{1}{2}-f}{f+g}\right)
\end{aligned}
$$

which is exactly of the required form with

$$
p_{k}=(-1)^{k} \frac{2^{k-1}}{\Gamma(1+k)} a_{k}
$$

The spectral functions for the complete solution of the colliding plane wave problem expressed in terms of these functions are therefore given by

$$
A(\sigma)=\sum_{k=0}^{\infty} p_{k}\left(\frac{1}{2}-\sigma\right)^{k}\left(\frac{1}{2}+\sigma\right)^{-1 / 2}, \quad B(\sigma)=\sum_{k=0}^{\infty} q_{k}\left(\frac{1}{2}-\sigma\right)^{k}\left(\frac{1}{2}+\sigma\right)^{-1 / 2},
$$

where $p_{0}=\sqrt{2 k_{+}}$and $q_{0}=\sqrt{2 k_{-}}$.

Remarkably, this provides a feasible way to solve the above characteristic initial value problem. It is not necessary to determine the two sets of constants $a_{k}$ and $b_{k}$ from the initial data functions $V_{+}(f)$ and $V_{-}(g)$. Rather, it is only necessary to determine the spectral functions from the initial data. The functions $A(\sigma) \sqrt{\frac{1}{2}+\sigma}$ and $B(\sigma) \sqrt{\frac{1}{2}+\sigma}$ should then be expanded as power series in $\frac{1}{2}-\sigma$. The resulting coefficients are exactly the coefficients $p_{k}$ and $q_{k}$ from which the coefficients $a_{k}$ and $b_{k}$ can be determined using (62) and the equivalent expression for $q_{k}$. In this way, the general solution (56) in the interaction region has been constructed explicitly, albeit in terms of a pair of infinite series. 


\section{Observations}

It has been demonstrated above that the initial value problem for colliding plane waves has been solved in principle for the vacuum case in which the approaching gravitational waves have constant and aligned polarization. The initial data are specified by two functions $h_{+}(u)$ and $h_{-}(v)$ which describe the profiles of the approaching waves. The first step is to solve the differential equations (3) for $P_{ \pm}$and $Q_{ \pm}$. From these, the functions $U_{+}(u)$ and $U_{-}(v)$ are determined, and this specifies the functions $f(u)$ and $g(v)$. They also determine the functions $V_{+}(u)$ and $V_{-}(v)$, and the next step is to construct the spectral data functions $A(\sigma)$ and $B(\sigma)$ using (31). From these, the final solution for $V(f, g)$ in the interaction region is obtained by evaluating the integrals in (29).

The method described above is correct in principle. In practice, however, difficulties will occur at several stages. The greatest difficulties in the construction of exact solutions will arise in the explicit evaluation of the integrals for $A(\sigma)$ and $B(\sigma)$ in (31), and then particularly in determining the final expression for $V(f, g)$ using (29). Also, although expressions for $f(u)$ and $g(v)$ are determined explicitly using the above procedure, it is not often that they can be inverted to construct $V_{+}(f)$ and $V_{-}(g)$. Nevertheless, it is possible to continue to use $u$ and $v$ as coordinates throughout the above calculations.

Of course, the above procedure could be used as the basis for a numerical solution of the problem. Indeed, for the collision of two pairs of impulsive waves or two sandwich waves, this may be the only viable option. For such an approach, care needs to be taken in evaluating the integrals, particularly near the limits as the integrands there behave as $|f-\sigma|^{-1 / 2}$, and $V_{+}^{\prime}\left(\frac{1}{2}\right)$ and $V_{-}^{\prime}\left(\frac{1}{2}\right)$ are also unbounded.

For the linear case considered above, the metric is diagonal and the solution is derived with the aid of the Abel transform. Unfortunately, this approach cannot be extended to the more general case in which the waves are not colinear. In the general case, the approaching gravitational waves either have variable or nonaligned polarization or electromagnetic wave components are included. In either case the main field equations are essentially nonlinear. However, the field equations are exactly the Ernst equations and their associated quadratures which are known to be integrable. Nevertheless, none of the known solution-generating techniques that are associated with these equations are adapted to initial data on a pair of null characteristics. An alternative approach has therefore been developed [10], [11]. However, further work is required to investigate whether or not this approach can deal in practice with realistic initial data.

\section{Acknowledgments}

For many useful discussions, the authors are particularly grateful to Professor G. A. Alekseev, whose collaboration on this topic was partially supported by the EPSRC and the INTAS grant 99-1782.

\section{References}

[1] Griffiths, J. B. (1991), Colliding plane waves in general relativity, (Oxford University Press).

[2] Szekeres, P. (1972), J. Math. Phys., 13, 286-294.

[3] Yurtsever, U. (1988), Phys. Rev. D, 38, 1706-1730.

[4] Hauser, I. and Ernst, F. J. (1989), J. Math. Phys., 30, 872-887.

[5] Hauser, I. and Ernst, F. J. (1989), J. Math. Phys., 30, 2322-2336.

[6] Hauser, I. and Ernst, F. J. (1990), J. Math. Phys., 31, 871-881. 
[7] Hauser, I. and Ernst, F. J. (1991), J. Math. Phys., 32, 198-209.

[8] Alekseev, G. A. (1985). Sov. Phys. Dokl., 30, 565-568. (Dokl. Acad. Nauk. SSSR, 283, $577-582,1985)$.

[9] Alekseev, G. A. (1987). Tr. Mat. Inst. Steklova, 176, 211; (Proc. Steklov Inst. Maths., 3, $215-262,1988)$.

[10] Alekseev, G. A. and Griffiths, J. B. (2001), Phys. Rev. Lett., 87, 221101.

[11] Alekseev, G. A. and Griffiths, J. B. (2002), in preparation.

[12] Khan, K. A. and Penrose, R. (1971), Nature, 229, 185.

[13] Ferrari, V. and Ibañez, J. (1988). Proc. Roy. Soc. A, 417, 417-431.

[14] Alekseev, G. A. and Griffiths, J. B. (1995), Phys. Rev. D, 52, 4497-4502.

[15] Alekseev, G. A. and Griffiths, J. B. (1996), Class. Quantum Grav., 13, 2191-2209.

[16] Alekseev, G. A. and Griffiths, J. B. (1997), Class. Quantum Grav., 14, 2869-2880. 